\title{
La profesionalización del sector de los cuidados
}

\section{Carolina Recio Cáceres*}

<carolina.recio@uab.cat>

\section{Sara Moreno-Colom*}

\section{Vicent Borràs Català*}

\section{Teresa Torns Martín*}

* Centre d'Estudis Sociològics sobre la Vida Quotidiana i el Treball, Institut d'Estudis del Treball, Universitat Autònoma de Barcelona

\begin{abstract}
Mendebaldeko gizarteen erronka nagusienetakoa da biztanleriaren zahartzea, eta horren ondorioz moldatu egin behar da zainketa sozialen antolatzeko modua. Zein gizarte-babes, nork edo nola bete behar dira zainketa-jarduera horiek? Galdera horien erantzun politikoa zehazten da zainketen izaera profesionala lortzeko modu bateko edo besteko sistema bat bideratzean. Artikulu honen xedea da Norberaren Autonomia Sustatzeko eta Mendetasunaren Arretarako Legea aintzat hartuz, epe luzeko zainketak profesional bilakatzeko prozesu horien mugak aztertzea. Oinarrizko hipotesiaren arabera, izaera sozial eta egiturazko faktore batzuek mugarri gertatzen dira kualifikatua eta laneko baldintza egokiak dituen enplegua sortzeko. Izaera kualitatiboa duen estrategia bat proposatzen da, eta oinarri hartzen da zaintzaileen profil desberdinak kontuan hartzen dituzten diskurtso eta imajinarioen azterketa burutzea. Bertan lortutako emaitzen arabera, zailtasunak nabarmentzen dira, eta jatorri izaten dituzte, batetik, gaur egungo profesionalen logika, eta, bestetik, Espainiako gizartean nagusi diren balio, ildo eta familia- eta kultura-tradizioak babesten dituzten imajinario kolektiboak.
\end{abstract}

\section{GAKO-HITZAK:}

Zahartzea, epe luzeko zainketak, imajinarioa, merkatu profesionalak, kualifikazioa.
El envejecimiento de la población es uno de los principales retos de las sociedades occidentales, que obliga a pensar cómo organizar socialmente los cuidados: ¿qué tipo de protección social, quién debe prestar los cuidados o cómo deben prestarse? La respuesta política a estas preguntas se traduce en un sistema u otro de profesionalizar el trabajo de cuidados. El objetivo de este artículo es analizar los límites del proceso de profesionalización de las ocupaciones vinculadas a los trabajos de cuidado de larga duración a partir de la Ley de Promoción de la Autonomía Personal y Atención a las personas en situación de Dependencia. La hipótesis principal apunta hacia la existencia de factores socioestructurales que limitarían las posibilidades de construcción de un empleo reconocido, cualificado y, por tanto, con buenas condiciones laborales. Se propone una estrategia metodológica cualitativa, basada en el análisis de los discursos y los imaginarios de distintos perfiles de personas cuidadoras. Los resultados obtenidos permiten constatar la existencia de dificultades que provienen de la lógica de las profesiones existentes y de los imaginarios colectivos que amparan los valores, pautas y tradiciones familiares y culturales hegemónicas en la sociedad española.

\section{Palabras Clave:}

Envejecimiento, cuidados de larga duración, imaginarios, mercados profesionales, cualificación. 


\section{Introducción}

Los cuidados de larga duración conforman el núcleo duro de las necesidades sociales surgidas con los cambios demográficos habidos en las sociedades del bienestar contemporáneas durante las tres últimas décadas. La razón primordial reside en el envejecimiento de la población que se encuentra limitada para desarrollar, de manera autónoma, las actividades cotidianas (Bettio et al., 2012). Una situación que parece haberse consensuado en torno al término dependencia. Tales factores generan un agujero demográfico, dada la paulatina desaparición de las mujeres de la "generación sándwich" (Miller, 1981; Williams, 2004¹). Pickard (2012) reconoce la existencia de este agujero demográfico en el conjunto de países europeos, bajo la idea de care gap. La literatura científica destaca cómo las mujeres de las familias han sido, y son hasta la fecha, las principales encargadas de gestionar, planificar y ejecutar las tareas de cuidados y atención a las personas dependientes de la familia. Este protagonismo femenino también puede observarse si se atiende a los datos de empleo en el subsector de servicios de cuidados, tanto en el ámbito público como en el privado (Torns et al., 2014).

El reto que supone el denominado care gap en las sociedades occidentales obliga a revisar la organización social de los cuidados. En este sentido, se plantean cuestiones sobre el tipo de protección social que deben ofrecer los Estados, sobre quién debe prestar los cuidados o sobre cómo deben prestarse. Las respuestas políticas a estas preguntas se traducen en distintos sistemas de profesionalización del trabajo de cuidados, básicamente porque, como argumentan distintas voces, el Estado y sus políticas sociales son elementos clave para entender los procesos a partir de los cuales los cuidados se profesionalizan (Henriksson, et al., 2006; Kuhlmann y Lynn, 2008). Desde esta perspectiva teórica, se plantea el análisis de los procesos de profesionalización de los cuidados de larga duración en España, atendiendo a sus características como Estado del bienestar y a las políticas impulsadas en el ámbito de los cuidados.

Con relación al modelo de Estado, tradicionalmente el régimen de cuidados español se ha considerado un ejemplo de familismo implícito, cuya cultura de los cuidados se centra en la familia y posee una protección social débil (Leitner, 2003; Saraceno, 2010). El peso familiar se complementa con una escasa asunción de responsabilidades domésticas por parte de los hombres y una presencia notoria de mujeres inmigradas como servicio doméstico que cubren, desde la informalidad, los huecos que dejan las mujeres de la familia (Parella, 2003; Martínez Buján 2011; Krüger y Jiménez 2013). Además, como

${ }^{1}$ Williams se refiere con esta expresión a las mujeres de la generación contigua, es decir, mujeres de 45 a 65 años que están cuidando simultáneamente de sus hijos/as y sus padres/madres. recuerda Saraceno (1995), a la informalidad familiar se le añade una visión asistencialista de los servicios públicos que se perciben como sustitutos de la familia y no como un derecho individual y universal.

Con relación a las políticas, a principios de la década de 2000 , en España surgen los primeros documentos y señales de alarma sobre el envejecimiento de la población. Prueba de ello es la publicación, en 2005, del Libro blanco de la dependencia. Se trata de un documento que, más allá de diagnosticar la situación, señalaba el incremento de un sector de población con necesidades de cuidado que no iban a ser resueltas a través del cuidado informal, el que habitualmente habían realizado las mujeres adultas de las familias. La población dependiente iba a necesitar unos cuidados sociosanitarios, en otras palabras, cuidados muy específicos debidos a su maltrecho estado de salud. Unos cuidados que, además, y en vistas del incremento de la esperanza de vida, podían prolongarse durante bastante tiempo. Los cuidados a las personas dependientes iban a requerir una expansión de los dispositivos públicos -o público-privados-y la creación de cuerpos de profesionales específicos, capacitados para dar una respuesta profesionalizada a las necesidades sociosanitarias que plantearían el aumento de usuarios dependientes.

A finales de 2006, se aprobó definitivamente la Ley de Promoción de la Autonomía Personal y Atención a las personas en situación de Dependencia (LAPAD). Este nuevo marco regulador se aprobó con el fin de consolidar un sistema estatal de servicios y prestaciones. Los cambios sociales y legislativos hacían prever un proceso de transición hacia un nuevo planteamiento de los servicios sociales y, en concreto, de los servicios orientados a la atención de las personas en situación de dependencia. Tal transición parecía estar destinada a la construcción de un sistema público universal y basado en cuidados profesionales. Sin embargo, las referencias de la LAPAD a la profesionalización eran bastante vagas y se limitaban a definir los cuidados profesionales como aquellos que no prestan miembros de la familia (artículo 2). Asimismo, en el apartado destinado a definir la calidad del servicio, se defendía la necesidad de avanzar en materia de calidad de empleo y en la definición de los requerimientos formativos (título II, artículos 35 y 36):

Cuidados profesionales: los prestados por una institución pública o entidad, con y sin ánimo de lucro, o profesional autónomo entre cuyas finalidades se encuentre la prestación de servicios a personas en situación de dependencia, ya sean en su hogar o en un centro. [Artículo 2. Definiciones]

Tomando como punto de partida la declaración de intenciones que incluye la LAPAD, el presente artículo analiza las dificultades y límites para profesionalizar las ocupaciones vinculadas a los cuidados de larga duración en España, ejemplo de régimen de 
cuidados familista ${ }^{2}$. Concretamente, se entiende que la profesionalización supone el reconocimiento y el prestigio de los saberes vinculados al cuidado $y$, por tanto, la necesidad de contar con personal adecuado y formado para satisfacer las necesidades de cuidados sociosanitarios. La hipótesis principal apunta la existencia de factores socioestructurales que limitarían las posibilidades de construcción de un empleo reconocido, cualificado y, por tanto, con buenas condiciones de empleo. Con el fin de analizar hasta qué punto dichos factores representan una dificultad para la profesionalización de los cuidados, se desarrolla una estrategia metodológica cualitativa, basada en el análisis de los discursos y los imaginarios de distintos perfiles de personas cuidadoras y personas expertas en el sector.

El artículo contiene tres apartados: el marco teórico, la descripción de la estrategia metodológica y la presentación de los resultados. Tras esbozar el estado de la cuestión sobre la discusión teórica que relaciona los conceptos profesión, cuidados y género, se presenta brevemente la investigación que sirve de base empírica para el artículo. El apartado de resultados señala las principales claves interpretativas del caso español, que se sintetizan en las conclusiones finales, recuperando la discusión teórica. En este sentido, se plantea la existencia de dificultades para profesionalizar los cuidados, dificultades debidas a la lógica de las profesiones existentes, así como de los imaginarios colectivos que amparan los valores, pautas y tradiciones familiares y culturales hegemónicas en la sociedad española.

\section{La profesionalización de los servicios de cuidados de larga duración}

\subsection{El desarrollo tardío de los sistemas de organización social del cuidado en Europa}

Kuhlmann y Lynn (2008) explican que la emergencia de proyectos profesionales está muy ligada al desarrollo de las sociedades modernas y los Estados del bienestar nacionales a finales del siglo XIX y principios del XX. Asimismo, defienden, junto con otras autoras, que la idea de las profesiones responde a proyectos de hombres blancos de clase media-alta con pretensiones de movilidad social ascendente (Davies, 1996). En esta misma línea, Henriksson (2006) recuerda como la noción de profesión es una construcción masculina que da forma a los proyectos profesionales. Asimismo, la investigación feminista subraya que el desarrollo del Estado del bienestar devalúa el trabajo de cuidados, dándole un espacio limitado en las políticas sociales (Julkunen, 1986; Rantalaiho, 1986, cit. en Henriksson, 2006).

${ }^{2}$ Se trata de un trabajo surgido en el marco de una investigación más amplia dirigida per la Dra. Teresa Torns Martín y financiada por el Instituto de la Mujer (PROFESOC, ref. 2011-0004-INV-00120).
Efectivamente, el modelo de bienestar, creado en Europa tras la Segunda Guerra Mundial, si bien produjo un gran volumen de empleo femenino, no siempre tuvo en cuenta todas las necesidades sociales que cubrir, ni se preocupó por la calidad de los empleos creados. En principio, las primeras políticas de bienestar, se enmarcaron en el ámbito de los servicios que pudieran dar cobertura a las necesidades de protección social de la población activa en el mercado laboral. Ello explicaría el desarrollo de las tres grandes áreas con servicios que, en la actualidad, dan cobertura universal en aquellos países donde el Estado del bienestar existe: pensiones, educación y sanidad. Tal cobertura, sin embargo, no tuvo en cuenta la variabilidad de las necesidades de cuidados y de bienestar de las personas en su vida cotidiana, a lo largo del ciclo de vida. Y si bien se aceptó que las necesidades cotidianas de cuidados eran perentorias al comienzo de la vida, no se previó que el alargamiento de ese ciclo de vida iba a cuestionar la bondad del modelo social europeo. Un modelo, hoy en crisis, que siempre tuvo como eje prioritario asegurar la producción de bienes y servicios, dando por supuesto que cualquier persona adulta podía afrontar el bienestar cotidiano por sí misma. Esta suposición enmascaró el hecho de que la gran mayoría de la población obtuviese y procurara ese bienestar cotidiano a través de intercambios más o menos equitativos de trabajo de cuidados, dinero, tiempo, amor o bienes en especie.

Así, no parece aventurado afirmar que este modelo social europeo siempre ha dado por supuesto que los cuidados y el bienestar cotidiano existen, pero no tienen por qué ser valorados o reconocidos. Una realidad a la que Carol Pateman (1995) se refiere como el contrato sexual sobre el cual, sin jamás citarlo o tenerlo en cuenta, se construyó el pacto social entre capital y trabajo. Como consecuencia, las mujeres son, de manera mayoritaria, quienes llevan a cabo el trabajo de cuidados, tanto en forma de servicios pagados como en su versión de trabajo familiar no pagado. Ello las convierte en las principales procuradoras del bienestar cotidiano. Borderías, Carrasco y Torns argumentan que esta situación "entre otras cosas deriva de una naturalización de los trabajos de cuidado, capaz de enmascarar la capacitación técnica que requieren las personas que trabajan en este tipo de servicios [...], que quedan así encerrados en un círculo vicioso que gira en torno al amor como única fuente de emoción, lo femenino, la falta de prestigio, la descualificación y, en consecuencia, los bajos salarios" (2011: 47). Las mismas autoras recuerdan que, con este modelo, la sociedad del bienestar refuerza las desigualdades de género, clase y etnia.

En este contexto, uno de los problemas para la organización social de los cuidados de larga duración reside en las dificultades para construir sistemas de atención sociosanitaria con una estructura profesional nítida. A partir de la literatura especializada, se identifican dos obstáculos para 
la profesionalización de los cuidados. En primer lugar, la dificultad de delimitar qué actividades se inscriben bajo el apéndice de empleo de cuidados (Christopherson, 1997; Cameron y Moss, 2007; Anxo y Fagan, 2005). Esta complejidad, vigente en buena parte de los países europeos, se traduce en una estructura profesional difusa. Los análisis realizados señalan la inexistencia de una profesión específica en el área de los cuidados de las personas consideradas como dependientes, a diferencia de lo que sucede en sectores como la salud y la educación, donde, ayudados por una mayor tradición histórica, sí es posible reconocer profesiones y categorías bien delimitadas. Por el contrario, y en segundo lugar, en el campo de la atención a las personas consideradas como dependientes existe sólo un conjunto difuso de profesiones o categorías profesionales caracterizadas por: falta de requerimientos formativos, baja cualificación, bajos salarios, escaso reconocimiento profesional y pocas posibilidades de desarrollar una trayectoria profesional ascendente (Johansson y Moss, 2004; Anxo y Fagan, 2005). Así pues, se trata de empleos precarios, de bajísima calidad y tan próximos al asistencialismo como alejados de unos servicios públicos profesionalizados y prestigiados 3 . Para entender este carácter desigual del trabajo de cuidados, es preciso considerar su vinculación con los saberes femeninos (Torns et al., 2014).

\subsection{Aproximaciones teóricas a la profesionalización de los cuidados}

Con el fin de analizar los límites existentes en la construcción de profesiones en torno a los cuidados, se propone una aproximación teórica compleja que incorpore como factores explicativos elementos materiales, simbólicos y culturales. La perspectiva tradicional de la sociología ha centrado sus análisis en la funcionalidad de las profesiones, sin problematizar ni su proceso de construcción social, ni las relaciones de poder que esconden en términos de jerarquías laborales y prestigio social (Henriksson, 2006). Desde esta aproximación, la subordinación de las ocupaciones de las mujeres en el sector de los cuidados se ha tratado como una consecuencia natural de la división del trabajo en el ámbito de la salud. Pero a partir de los años setenta, surgen propuestas críticas que subrayan desde el carácter histórico de las profesiones hasta las relaciones de dominación y monopolio que existen dentro de los grupos profesionales. Distintos análisis sobre los procesos de construcción de las profesiones señalan la importancia de los Estados y sus políticas, así como el peso que juega la clase social, el género y la etnia. Más concretamente, la investigación feminista evidencia cómo los privilegios profesionales de los grupos masculinos se institucionalizan a través

3 En el caso español, la complicidad de la Administración en el desprestigio de estos empleos se evidencia en el Decreto 1424/1985, de 1 de agosto, por el que se regula la relación laboral de carácter especial del servicio del hogar familiar, una norma laboral que durante muchos años, hasta la aprobación de la LAPAD y el posterior Decreto $1620 / 2011$, permitió prácticas abusivas y discriminatorias. del Estado, en un proceso donde se marginaliza el trabajo de las mujeres. Además, los estudios comparativos ponen de manifiesto cómo las variaciones nacionales en los grupos profesionales y las relaciones de género dan lugar a distintos patrones de segregación ocupacional (Crompton y Le Feuvre, 2003). Estos estudios sugieren realizar análisis multinivel de los procesos de género que se dan en las instituciones sociales que representan las profesiones (Henriksson, 2006).

Tomando como punto de partida estas reflexiones, desde la sociología del trabajo y de las profesiones con perspectiva de género se plantean algunas cuestiones sobre las dificultades que limitan el avance hacia sistemas de cuidados profesionalizados. A continuación, se detallan tres aproximaciones consideradas por su potencial explicativo, centrado en los mercados profesionales, la construcción social de las cualificaciones y las relaciones de poder, respectivamente.

\subsubsection{Cuidados y construcción de mercados profesionales}

Friedson construyó el concepto de profesión interrelacionando tres dimensiones: la visión durkheimiana de moralidad y desempeño de una profesión; la visión de Talcott Parsons sobre el conocimiento experto; la visión de Abbot sobre la jurisprudencia que diferencia una profesión de otra. En este sentido, defendía que el paso de una actividad laboral a una profesión reconocida depende del rol/estatus de los sistemas de formación histórica y territorialmente contextualizados, de la acción de las asociaciones profesionales y de las posibilidades para generar un segmento del mercado relativamente protegido, mediante el requerimiento de certificados o licencias (Friedson, cit. en Gadrey, 1994). Esta definición es válida para profesiones consolidadas y bien valoradas, como por ejemplo, la profesión médica o la abogacía. Ello implica contar con una acción firme de grupos profesionales y trabajadores que defiendan y definan la profesión. Aquellos empleos considerados altamente cualificados tienen mayores probabilidades de estar en los segmentos laborales de mayor prestigio social, mejores condiciones salariales y convertirse en mercados profesionales (Recio, 1997).

En la medida que las actividades de cuidados no se encuentra entre estos segmentos cualificados, se articulan otras propuestas teóricas capaces de combinar el conocimiento experto sobre el cuidado con una atención personalizada de las personas usuarias. La profesión de cuidar implica generar y consolidar sistemas de cualificaciones, credenciales profesionales, códigos éticos y normativas específicas, y al mismo tiempo, no se puede perder de vista que las personas usuarias deben tener reconocido el derecho a decidir cómo quieren ser cuidados. Como crítica a Friedson, Knijn y Verhagen (2007) conciben la profesionalidad en el área de los 
cuidados como un escenario nuevo y distinto, sujeto a tres lógicas para organizar y pensar el empleo. En primer lugar, la lógica del Estado, que parte de la idea de igualdad para definir las obligaciones y los derechos de ciudadanía sobre el cuidado y diseñar una red de servicios y prestaciones. En segundo lugar, la lógica del mercado, que no parte de una idea de igualdad, sino que se basa en la elección privada de los servicios de cuidado, que se convierten en productos intercambiables bajo el criterio del máximo beneficio. Y en tercer lugar, la lógica de la familia, que se asienta sobre un concepto de privacidad sensiblemente distinta del carácter privado de la lógica de mercado, erigiéndose sobre conceptos como emotividad, intimidad, solidaridad, empatía, reciprocidad. Las autoras citadas defienden que la profesionalización de los cuidados se debe construir sobre la base del conocimiento experto, y no sobre la vinculación del empleo con las tareas del hogar realizadas por mujeres (Duyvendak et al., 2005; Knijn y Verhagen, 2007).

\subsubsection{Cuidados, segmentación del mercado de trabajo y construcción social de la cualificación}

Partiendo de los planteamientos sobre la segmentación del mercado de trabajo, otros estudios en relación con los empleos en el sector de los cuidados plantean una crítica a la construcción supuestamente neutra de las cualificaciones. En gran parte, estos trabajos provienen de la tradición francesa de los estudios de la cualificación, incorporado consideraciones al análisis específico de los empleos del cuidado (Ribault, 2008 y 2009; Jany Catrice et al., 2009; Devetter et al., 2008). Estos autores cuestionan la idea de profesión, al tratarse de un concepto que justifica una posición jerárquica de una actividad sobre otra. La profesionalización sería una construcción ideológica basada en la limitación de las puertas de entrada y en la adquisición de una posición de poder respecto a otro grupo profesional, promoviendo la individualización de las relaciones laborales.

En general, estos autores centran sus críticas sobre los procesos habituales de construcción de una profesión de prestigio, porque difícilmente se adecuan a los empleos de cuidado. Los parámetros habituales para medir las profesiones no consideran los aspectos específicos de estos empleos que escapan a ciertas formas de medición de la cualificación profesional. Esto implica la justificación que aquellos trabajos sin valor social tengan pésimas condiciones de empleo y se sitúen en los últimos puestos de la cadena clasificatoria de los empleos (Jany-Catrice et al., 2009). En este sentido, recuerdan que los empleos del cuidado se entienden como la externalización del trabajo doméstico y familiar, un campo sin valor social y con muchas barreras para construirse como profesión. Estas posiciones recuerdan que la profesionalización requiere un proceso de racionalización y estandarización de las tareas, pero advierten que este circuito profesional, válido para un empleo industrial, no se adecua a las economías donde predominan las actividades del sector terciario (Devetter y Rosseau, 2007; Dussuet, 2002 y 2005; Ribault, 2008 y 2009). En esta línea argumental, se defiende que el proceso de mejora del empleo, y si se quiere, de profesionalización, no se nutre sólo de las acciones realizadas en el mercado de trabajo. Se trata de empleos demasiado vinculados a los imaginarios del trabajo doméstico y familiar. Una mejora de los empleos debe ir ligada a un cambio de los imaginarios y las prácticas sociales sobre el trabajo doméstico y familiar. Una tarea difícil, porque como Fraisse (2000) advertía, resulta muy difícil dotar de valor una actividad que socialmente se asocia a la privacidad, a la domesticidad y a la servidumbre.

\subsubsection{Cuidados, profesionalización y relaciones de poder}

Más allá de la construcción de los mercados profesionales y las cualificaciones, otro conjunto de análisis tratan de explicar por qué hay ocupaciones, dentro del área del cuidado, que han conseguido cristalizarse como profesiones y otras que no. Se trata de una visión distinta sobre la construcción de profesiones, interesada en explicar cómo las relaciones de poder condicionan el significado del cuidado que se asocia a ciertas tareas y, en función de él, se reconoce o no como un conocimiento profesional (Hugman, 1991). El poder de los grupos sociales para intervenir en la determinación de los tipos de profesionales es un elemento central en la institucionalización de una profesión (Evertsson, 2000; Hugman, 1991). La capacidad para consolidar una profesión cualificada y reconocida socialmente también depende de la capacidad de presión de los actores sociales en las instancias políticas y económicas, sobre todo teniendo en cuenta el papel del Estado en el control y definición de las profesiones (Hugman, 1991). En este sentido, Abbot (1988) defiende que la consolidación e institucionalización de las profesiones en el área del cuidado se determina de dos formas: por un lado, el tipo de relación entre el empleo y el trabajo doméstico y familiar; por el otro lado, el contexto político y económico, que influye en el peso de los actores sociales para negociar y delimitar el estatus de estas profesiones. La historia de profesiones similares, siendo la enfermería el ejemplo paradigmático, pone de relieve una estrategia de acciones de poder por parte de grupos de presión, que consiguen así establecer mecanismos para limitar la entrada de personal y la adquisición de privilegios profesionales (salarios, reconocimiento). En este sentido, estudios recientes, como el de Hebson et al. (2015), apuntan la relación entre género y clase social para explicar el empleo de las trabajadoras de la atención domiciliaria en el Reino Unido. A través del concepto de habitus de Bourdieu, explican que no sólo el género explica la poca calidad de dichos empleos, también el origen de clase y la cultura de clase permiten entender que determinados 
colectivos de mujeres acepten y vivan como satisfactorios empleos de muy baja calidad. En el caso de la atención domiciliaria, las difusas fronteras entre el empleo remunerado y el no remunerado se suman a las dificultades para realizar un trabajo muy adscrito socialmente al espacio privado. Todo ello condiciona las relaciones interpersonales y contribuye a la invisibilización de los cuidados como un trabajo de las mujeres.

\section{Metodología 4}

El principal objetivo de la estrategia metodológica ha sido desbrozar y hacer emerger las dificultades materiales, simbólicas y culturales que permanecen en el imaginario de la sociedad española a la hora de vehicular unos servicios de cuidados de calidad, profesionalizados y dotados de reconocimiento social y económico. Para ello, se ha optado por una estrategia cualitativa con el fin de captary analizar las expectativas, las representaciones, las preferencias, las exigencias y los discursos que las personas con responsabilidades formales o informales de cuidados tienen en relación con el trabajo que desempañan como cuidadoras. Los discursos de las personas trabajadoras y de las mujeres cuidadoras en la familia permiten visibilizar las limitaciones de profesionalización de estos cuidados. Esos discursos muestran los imaginarios sobre el concepto de cuidar y cómo lo perciben los distintos actores implicados en el ámbito del cuidado.

En concreto, el trabajo de campo ha contemplado tres subfases, en función de la composición del universo de informantes analizado y el objetivo perseguido. En las dos primeras, se han realizado entrevistas en profundidad y grupos de discusión a distintos colectivos implicados en el cuidado de larga duración y en su organización social. En todos los casos, se ha limitado el universo de análisis a las mujeres, dado el carácter femenino de las cuidadoras tanto formales como informales. Para la selección de las personas, se ha utilizado la estrategia de bola de nieve, junto con la captación profesional. Todas las entrevistas y grupos de discusión se han transcrito literalmente para realizar el análisis de contenido con el soporte del software Atlas.ti.

Concretamente, durante la primera fase se han realizado 25 entrevistas en profundidad a empleadas en el sector de atención a las personas, en función del espacio donde prestaban el cuidado (hogar o institución), la relación contractual (formal o informal) y el lugar dentro de la estructura ocupacional del

4 La base empírica del presente artículo presenta una parte de los resultados obtenidos en el trabajo de campo del proyecto PROFESOC (ref. 2011-0004-INV-00120). El objetivo general de esta investigación ha sido analizar las posibilidades de profesionalizar los trabajos vinculados a la atención y los cuidados de larga duración; y entre los objetivos específicos, han estado el análisis de las dificultades y limitaciones de la profesionalización. cuidado). El objetivo de la entrevista ha sido captar los imaginarios a partir de la trayectoria laboral de las empleadas. Durante la segunda fase, se han realizado 8 grupos de discusión con cuidadoras familiares. El perfil de los grupos de discusión era el de mujeres mayores de 45 años que ejercían el rol de principal cuidadora en la familia. Se ha definido una tipología considerando el origen socioeconómico, el tipo de cargas de cuidado y la variable generaciónciclo de vida del universo de análisis. En concreto, se consideró necesario diferenciar las mujeres cuidadoras de la familia según sus condiciones materiales (situación laboral y nivel de estudios) y la etapa donde se encuentran desde la perspectiva del ciclo vital (generación sándwich 45-65 años y más de 65 años). El objetivo del grupo de discusión ha sido la captación de los imaginarios colectivos hegemónicos, confrontados con una misma situación vital. Finalmente, en la tercera fase se han realizado entrevistas semiestructuradas a profesionales, con el fin de discutir los resultados del trabajo de campo previo y avanzar en la construcción de propuestas para la profesionalización del trabajo de cuidados 5 .

\section{Resultados}

A partir de los planteamientos teóricos expuestos, se presentan los resultados identificando dos bloques importantes de dificultades para la profesionalización de los cuidados. El primero se refiere al contenido y los significados atribuidos a los cuidados. Se trata de dificultades que están en sintonía con las aproximaciones que, desde la sociología del trabajo, han señalado la importancia de los imaginarios sociales sobre el cuidado, situándolo más cerca de las relaciones serviles que del desempeño profesional. El segundo bloque tiene que ver con las limitaciones propias de la construcción de una estructura profesional específica. En este caso, son limitaciones relacionadas con el proceso de construcción de los empleos, donde es fundamental analizar cómo se prestigian los saberes que pueden generar la consolidación de un segmento profesional.

\subsection{Los cuidados: contenido, valor e imaginarios}

Uno de los principales límites para la profesionalización debe buscarse en la propia indefinición de lo que implica cuidar y del significado que las distintas personas entrevistadas dan al trabajo de cuidados. Esta dificultad viene dada por la existencia de una multiplicidad de maneras, espacios y condiciones de trabajo según los condicionantes específicos que los cuidados requieren. El consenso sobre los aspectos básicos que implican los cuidados, o dicho de otro modo, los indicadores
5 La primera fase del trabajo de campo se realizó durante entre enero y abril de 2012 , la segunda fase se desarrolló entre abril y julio de 2012, y la tercera fase se llevó a cabo entre septiembre y diciembre de 2012. 
principales sobre los cuales se basan las exigencias de familiares y de los propios trabajadores son tres: la higiene personal y del espacio; la alimentación; y los aspectos relacionados con la medicalización (administración de medicamentos, curas de heridas o llagas, entre otros). Si bien es cierto que son exigencias muy básicas, apuntan claramente cuáles son los estándares sobre los que se configura la exigencia social sobre un sistema de cuidados. No obstante, pueden variar en función de factores como la clase social de los dependientes, la tradición y experiencia de las instituciones que ofrecen los servicios de cuidado, los espacios donde se proporciona los cuidados, y los perfiles y condiciones laborales de las trabajadoras:

El tema de la higiene. Yo creo que todo, porque hay gente que se queja por la higiene, el orden, la alimentación. (Trabajadora Centro Residencial, atención directa)

Aquí estás cuidando a una persona, le estás dando de comer, lo estás duchando, lo estás trayendo al lavabo y limpiando, le estás cambiando los pañales. (Trabajadora Centro de Día, gestora)

Estar pendiente de la persona que está enferma: que esté bien aseado, curado, con sus pastillas y medicinas. que [las] hayan tomado puntualmente, como debe ser. (Empleada en el hogar)

Más allá de este consenso sobre los aspectos básicos que implican los cuidados, es interesante subrayar que el discurso de las empleadas del hogar incluye referencias al trabajo doméstico, una realidad ausente e invisible en el discurso de las trabajadoras en los centros. En este sentido, se observa cómo el espacio donde se realizan los cuidados puede condicionar tanto las tareas que se han de realizar como la consideración de éstas, siendo la dicotomía público/privado una dimensión clave para entender si la responsabilidad de los cuidados también lleva aparejada el trabajo doméstico.

\subsection{Un trabajo sin valor social y sobrevenido}

Las características propias de los cuidados, más allá de las condiciones laborales en las que se realizan, hacen que prácticamente todas las trabajadoras consideren que es un trabajo muy duro. Esta consideración se da, sobre todo, las que dedican muchas horas a esa actividad, fundamentalmente las que trabajan en centros residenciales o las que trabajan en los domicilios particulares, donde se da una sobrecarga continua de trabajo, en muchos casos sin apenas descansos. Esta situación contrasta con la de las trabajadoras de los servicios de atención domiciliaria (SAD), donde el hecho de realizar servicios a domicilio, con usuarios diferentes y cambios cada hora, les descarga de la presión que supone el cuidado ininterrumpido. Con todo, las trabajadoras coinciden en que la situación de deterioro físico y mental de los dependientes es lo que confiere más dureza y capacidad de desgaste al trabajo que realizan. Todas son conscientes de que necesitarían formación, recursos y, sobre todo, algún tipo de soporte para poder sobrellevar emocionalmente lo que representa su cotidianidad como cuidadoras. Significativamente, su discurso hace más hincapié en la necesidad de tener acceso a "vías de escape" de la carga que les supone el trabajo que en la importancia de la formación reglada para la consolidación de la profesión:

Yo creo que necesitan una preparación, no sé unas charlas o unas clases de pilates, o de yoga (ríe), algo durante la vida laboral, porque no se puede vivir así. Yo les veo las caras a algunas y no se puede vivir así toda la vida, es dañino para uno mismo o los demás. Es lo que yo haría para el trabajo, porque si tú estás bien, está bien todo lo otro. (Trabajadora Centro Residencial, gestora).

Otro de los consensos que verbalizan aquellas trabajadoras que más horas dedican, y cuyas condiciones laborales son más precarias y de mayor sumisión, es la consciencia de que la labor que realizan es muy importante, pero que nadie la valora:

Yo encuentro que esto no está pagado ni con todo el oro del mundo, a veces haces cosas, que digo: “¡Dios mío, quién te valora esto! Eres tú y lo que haces, sin esperar nada a cambio. Una nómina no es lo bastante, no es lo que cobras.

A veces me dicen "yo te pago, tienes que hacerme lo que quiera yo". Es una misma [la] que tiene que decir y valorarse que lo que hace está bien, porque si tienes que esperar que alguno te lo diga... No lo sé, yo no sé si los familiares son conscientes de las cosas que una llega a hacer por sus familiares que están allí. (Trabajadora Centro Residencial, atención directa)

El trabajo de cuidados no está valorado ni por las familias que lo necesitan, ni por los propios usuarios que lo reciben, lo que dificulta sobremanera que sea reconocido socialmente como un derecho de ciudadanía. Incluso para las mismas instituciones que ofrecen el servicio, el discurso siempre se vincula a la familia como principal responsable del cuidado, y por tanto, quien debe realizar la principal aportación:

Mi trabajo está muy poco valorado, muy poco. Poquísimo, nada. De hecho, la gente se piensa que esto es gratis, ¿vale? Que por el hecho de que sea una persona mayor, ya no tienen que pagar nada. Perdona, aquí estamos muchas horas, son 11 horas diarias. Profesionales. Gente que está trabajando para que tu padre o tu madre estén bien. Que es muy difícil llevar un grupo de gente que... Pero no lo valoran, no lo valoran. ¡Ah! Se piensan que ¡hala!, ¡que están allí asentados y ya está! ¿Sabes? Y no. ¡Hay todo un seguimiento de trabajo importante! (Trabajadora Centro de Día, gestora) 
Esta ausencia o baja valoración del trabajo de cuidados obedece, en cierta media, a un discurso social que se ampara en la percepción sobre el carácter sobrevenido e individual de este tipo de cuidado. A diferencia de lo que sucede con los hijos, los cuidados de los mayores no se planifican, aparecen sin estar preparado para ello y se perciben como un problema individual que depende de cada familia. La lógica general argumenta bajo un solo supuesto: "me ha tocado y lo debo solucionar como pueda". En este sentido, los cuidados aparecen como un objetivo en sí mismo, que condiciona la vida de los cuidadores, lejos de pensarse como una necesidad social que, en mayor o menor medida, afecta a todos y, por lo tanto, que demanda respuestas políticas en términos de voluntades y recursos.

\subsection{Hogar e individualización}

El análisis de las entrevistas y los grupos de discusión también pone de manifiesto las preferencias de las personas por el hogar familiar como el espacio del cuidado, dejando la institución como una opción residual y tan sólo válida para situaciones de gran dependencia. El hogar de la persona usuaria emerge como el escenario principal, ya sea porque se realiza un cuidado por parte de las mujeres de la propia familia, ya sea porque se contrata formal o informalmente personal de apoyo en el hogar. Esta centralidad del hogar familiar conlleva la preferencia por la individualización de los cuidados, al facilitar las elecciones sobre cómo y cuándo se prestan. A menudo, emerge de los discursos que una buena atención es aquella que permite ofrecer una atención individualizada a cada usuario. Este ideal iría a contracorriente de la lógica de la institucionalización, donde la organización del trabajo suele parcelar mucho las tareas de las profesionales y limitar los tiempos de atención directa. En este sentido, los trabajos que se realizan en los centros de día y residencias están fuertemente "rutinizados" y obedecen a una lógica cuasi industrial-fordista de distribución de tareas en tiempos que dificulta la atención individualizada del cuidado, y limita la autonomía de la trabajadora para decidir cuestiones importantes sobre su propio trabajo. Un tiempo disponible que además está relacionado con el número de personas que hay que cuidar y con el tamaño de las plantillas de profesionales del centro, dos elementos que pueden variar según el centro de trabajo y que tienen una incidencia importante en cuanto a los ritmos y cargas de trabajo.

A las nueve de la noche, y... bueno, yo llego, me visto y dejo mis cosas abajo, pongo una lavadora - porque nos toca hacer lavandería al turno de noche- y me subo arriba. Acuesto a la gente... Una vez acostados, acto seguido me pongo a revisarle el cambio pañal, porque no todo el mundo trabaja en condiciones para que estén tres horas bien, por eso yo prefiero revisarlo y ponerlo en condiciones. Entonces, también nos toca hacer el comedor donde desayunan todos: barrer, fregar las mesas, montar los servicios... Ahí se pasa unas dos horas y media ya. Luego me preparo el carro de la medicación nocturna que se da a las doce a los que toman sus pastillas o a los que quieren un vaso de leche, al que es diabético unas gotas, lo que haga falta. Hago otra ronda de una hora y media en repartir, más o menos. Serán casi la una. Dejó el carro y otra vez, otra ronda para mirar los pañales, y en cada ronda, cambio de postura al residente (Trabajadora Centro Residencial).

En cambio, los trabajos realizados en el propio hogar, ya sea por cuidadoras de la atención domiciliaria 0 por cuidadoras contratadas de manera más o menos formal, permiten una mayor individualización. La clave fundamentalmente radica en que hay un tiempo en que tanto el cuidador como el dependiente saben que se estará "pendiente de ellos", un tiempo en el que se atiende una necesidad específica de un único usuario. Este eje de la personalización del cuidado frente a la impersonalidad de éste en la institución aparece conectado con las relaciones de mayor o menor poder que se establecen entre persona cuidada y persona cuidadora (o entre familiar de la persona cuidada y persona cuidadora). El ideal del hogar familiar apunta a la percepción de un espacio donde se respeta la individualidad e intimidad del dependiente, al mismo tiempo que permite conservar el poder de decisión de cómo, cuándo y de qué manera se lleven a cabo las tareas de cuidado. Si bien desde una perspectiva de la profesión, la centralidad del hogar en los cuidados contribuye a su devaluación en la medida que, a menudo, implica aceptar la informalidad, la precariedad y la no profesionalización. A pesar de que en las entrevistas se reconoce que las trabajadoras informales realizan tareas especializadas, los requerimientos formativos brillan por su ausencia; tanto, que se puede recurrir a contradicciones en los discursos que, por un lado, apelan a la calidad del cuidado en el hogar en comparación a los centros residenciales, pero que, sin embargo, aceptan trabajadoras en casa sin conocimientos específicos. La contratación de mujeres inmigrantes apenas establece unas exigencias sobre qué saberes deberían tener las personas contratadas, y se asume contratarlas en condiciones muy precarias, como si esos aspectos no intervinieran también en la calidad del cuidado.

\subsection{Estructuras profesionales y cualificaciones}

Los cuidados de larga duración son un trabajo sucio, poco visible, que tan sólo van a poder realizar aquellas mujeres para las que este empleo es un proyecto laboral y no un proyecto profesional. Se han visto las connotaciones simbólicas que tiene el trabajo de cuidados de larga duración, se ha visto cómo lo viven y lo perciben las personas usuarias y las familias: se trata de un trabajo duro que apenas nadie reconoce. Sobre esta base se configuran los empleos y las profesiones vinculadas a este tipo 
de trabajo. Son cualificaciones y estructuras que dificultan que el trabajo de cuidados cotidiano y continuado sea considerado un trabajo prestigiado. Sí pueden serlo otro tipo de tareas vinculadas al trabajo de cuidados de larga duración, pero más alejadas de esa atención directa y cotidiana. Esto significa que se marcan líneas divisorias entre las trabajadoras. Las distinciones entre profesionales, aunque aparentemente neutras, en la práctica esconden una estructura muy jerarquizada que evidencia una desigual distribución de los prestigios, una estructura que pone de relieve el menor valor de las tareas de cuidado cotidiano, continuado y no médico. Se visibiliza en los discursos de las propias trabajadoras, y de las personas expertas, la aceptación de la menor valoración del trabajo de cuidados más próximo a la persona usuaria, aquellos que llamamos “trabajos de atención directa”.

\subsubsection{Perfiles y jerarquías profesionales}

Las trayectorias laborales y las posiciones de clase social son distintas entre las trabajadoras en el sector de cuidados de larga duración. En este sentido, se identifican tres perfiles: mujeres inmigrantes como cuidadoras informales, mujeres autóctonas de clase obrera como trabajadoras de SAD, mujeres autóctonas jóvenes como trabajadoras sociales.

La informalidad está reservada para mujeres inmigrantes que, sin tener experiencia en el sector, entienden que ésa es la única vía de entrada al empleo en el país receptor. Su proyecto laboral no es profesionalizador, sino de pura subsistencia o, en el mejor de los casos, una estrategia para conseguir la regularización:

Yo me vine aquí porque yo estaba en una buena empresa de allá, de Ecuador. Trabajé como quince años en una empresa, quince años, y esa empresa liquidó. Y después, como a mí me gustaba trabajar y ser independiente... Me gustaba tener, que yo, que yo trabaje, desde luego ayudarlo también. Y, entonces, ya ahí, en ese tiempo, ya, como son los hombres allá, que andan con la una, con la otra, y nos separamos, nos divorciamos. Y yo cogí mi rumbo y se me presentó una oportunidad de venirme, y me vine, solita. Me aventuré sola. Y llegué por primera vez a Málaga. Allá fue mi primera experiencia que tuve yo de un trabajo. También, una pareja de esposos: un médico forense y ella una abogada, y dos niños. Allí estuve trabajando como tres años. (Empleada en el hogar)

En el caso de las trabajadoras de atención directa del SAD, su trayectoria es algo distinta, puesto que la mayoría son mujeres autóctonas de clase obrera, con experiencia laboral, aunque sea en otros sectores, y a las que la necesidad de trabajar las ha ido conduciendo a un sector con cierta estabilidad en el empleo femenino. La necesidad y voluntad de estar presentes en el mercado de trabajo es su "proyecto laboral de vida". Suelen expresar cierta satisfacción con su empleo, porque saben que están generando bienestar a otras personas. Asimismo, asumen con naturalidad su precariedad, en parte por su posición de clase social y por el "escaso" valor que ellas mismas dan a las tareas de cuidado. Tienen experiencia laboral y hábitos laborales, su trayectoria vital pasa por el trabajo en el mercado de trabajo, compartido con su trabajo de cuidadoras en sus propios hogares. Entre las mujeres entrevistadas, abundan las historias de vida que relatan cómo, antes de la entrada en el sector de los cuidados, habían abandonado el mercado de trabajo para cuidar a familiares a su cargo. Esta experiencia informal que acumula la mayoría de ellas al tener familiares cercanos a su cargo es percibida como un saber necesario para poder desempeñar mejor su trabajo:

Yo trabajé 36 años en una empresa de cerámica aquí, en Cerdanyola. Empecé con 14 años. Había estudiado, tenía bastante titulación para poder trabajar en otro trabajo, pero me gustó la cerámica, la artesanía. Entonces, como era una empresa y estábamos todo mujeres y, bueno, había alguno que otro hombre, pero, bueno, y había buen ambiente., eso me encantó. Total, que duré 36 años, el tiempo [hasta] que la empresa cerró. Entonces, yo no quería seguir trabajando en una empresa, porque acabé harta de todo el día encerrada. Entonces, oí hablar de este trabajo, y hicimos un cursillo en El Safareig, aquí, en Cerdanyola..., que era todo de mujeres, que estabas en el paro. (Trabajadora SAD, atención directa)

Me quedé en paro, como quien dice. Entonces mi suegra enfermó, de cáncer, lo típico, estas cosas que pasan. Era ya en el ochenta y algo, cuando empezábamos a ser ricos, y entonces yo ya estuve sin trabajar como 10 años o así. (Trabajadora Centro de Día, atención directa)

Finalmente, las trabajadoras sociales son las encargadas de gestionar los servicios (determinar horarios, distribuir tareas) y coordinar a las trabajadoras auxiliares, tanto en las residencias como en el SAD. La mayoría, y a diferencia de las otras trabajadoras, son mujeres autóctonas jóvenes que acceden a este empleo como parte de su trayectoria profesional. Tienen titulación universitaria y se perciben como las profesionales de los servicios sociales, una distinción que refuerza el desprestigio sobre aquellos trabajos más manuales y cotidianos:

Hombre, siempre. Siempre, siempre, cuando la directora dice algo, es la palabra que queda, ¿vale?, es la palabra que queda. Y después, siempre, el profesional del equipo interdisciplinar siempre también tendrá. Su palabra tendrá más peso que la de las auxiliares, porque al fin y al cabo, es quien hace su terapia y es quien trae su grupo. Pero siempre, siempre, damos la oportunidad [de] que todo el mundo haga su valoración. (Trabajadora Centro Residencial, gestora) 
La diferencia no se establece sólo entre perfiles profesionales: estas figuras laborales también desempeñan sus tareas en espacios distintos. El espacio en el que se desarrolla el cuidado interviene en la forma de organizarlo y en las exigencias de su prestación. Cuando el hogar es protagonista, se tiende a observar relaciones casi de servilismo y esclavitud, aunque la persona que está contratada (formal o informalmente) destaque la importancia de generar vínculos emocionales o amorosos, de carácter casi familiar. En el caso que los cuidados se realicen en una institución, el contenido del trabajo es distinto, está mucho más pautado, tanto, que a menudo los discursos de los procesos de trabajo recuerdan a la típica cadena de montaje de una empresa fordista. En ese caso, las relaciones entre personas implicadas en el proceso de cuidar se expresan de forma distinta, y es ahí donde, se torna evidente el escaso valor de la atención directa, visibilizándose la estructura jerárquica de las profesiones vinculadas al trabajo de cuidados.

En efecto, además de la estructura profesional, resulta relevante constatar la existencia de una jerarquía profesional clara que facilita la persistencia de la no profesionalización de los trabajos de cuidados. La banalización e invisibilidad de los cuidados justifican la diferencia entre las tareas de atención continuada y la gestión de los cuidados. El imaginario del profesional, en particular el de aquellas personas que atienden directa y cotidianamente a la persona dependiente, es el de llevar a cabo un trabajo sucio, poco visible, que tan sólo van a poder realizar aquellas mujeres que se conforman, o se ven atrapadas, en una actividad laboral, sin aspiraciones profesionales. Estas connotaciones impiden que las tareas básicas puedan alcanzar un mayor prestigio. Sin embargo, sí parece que pueden prestigiarse otro tipo de tareas vinculadas al contenido de los servicios de cuidados de larga duración, cuando quedan alejadas de esa atención directa y cotidiana. Esta situación, no obstante, supone la existencia de una estructura jerárquica de las profesionales implicadas alrededor de los cuidados de las personas dependientes, la cual, a su vez, esconde una desigual distribución de poderes y prestigios. El último escalón de esta jerarquía siempre está ocupado por quienes se dedican a las tareas cotidianas de unos cuidados continuados, salvo los de carácter médico. En esos casos, tan sólo se consideran con rango profesional las tareas y actividades relacionadas con la gestión y organización de los cuidados o los cuidados no cotidianos. Una realidad que, probablemente, tiene mucho que ver con el proceso no neutral, desde el punto de vista de género, clase y etnia, de la construcción de las cualificaciones profesionales.

\subsubsection{Los saberes: invisibilidad de las cualificaciones y naturalización de las competencias}

Las entrevistas explican que los saberes necesarios para realizar los trabajos de cuidado continuado requieren actitudes y aptitudes específicas que, en caso de no tener, implica no saber hacer esa tarea. Sin embargo, estas actitudes y aptitudes a menudo no están suficientemente reconocidas, de manera que los saberes relacionados con el trabajo de cuidados están desprestigiados. Este desprestigio contribuye a invisibilizar conocimientos y a naturalizar competencias.

Desde el punto de vista de la invisibilidad de cualificaciones, entendida como forma de no reconocimiento de los conocimientos, en los discursos analizados se detectan dos ideas que aparecen como complementarias y contradictorias. En primer lugar, se observa que aquello que delimita un buen trabajo en este ámbito está muy relacionado con competencias actitudinales, de saber estar. Estos elementos que pueblan el campo de lo subjetivo suelen tener una difícil traducción en modo de capacitación técnica. Es decir, escapan a la lógica dominante de construcción de la cualificación, pero sin embargo, se entienden como imprescindibles para el buen desempeño de la profesión. De hecho, la experiencia no laboral se toma en cuenta: los años de vida necesarios para realizar este empleo se consideran un elemento positivo para el desempeño de la actividad. El problema es que no se convierten en competencias educativas evaluables. La experiencia de vida, entendida como los aprendizajes realizados por estas mujeres en su vida privada, no son tomados en consideración, aunque todos los actores los consideran aprendizajes necesarios para la realización de este trabajo:

Mira, las auxiliares, desde mi punto de vista, tienen que ser personas con un bagaje personal importante, que sepan traer una familia. ¿Y por qué te lo digo, esto? Porque, mira, las abuelas tienen su picaresca, y quieren que le rías las gracias y quieren que te las ganes. Y las chicas, si son un poco listas, se las sabrán ganar. Aunque te caiga bien o mal, pero tú sabrás traértela, ¿me entiendes? En cambio, una chica que tiene poca, no sé, poca; [que sea] muy poco experimentada en la vida, seguramente, pues, se le pondrá de culo a la abuela, no le reirá las gracias, si la abuela se queja, le pondrá mala cara, etcétera, etcétera, etcétera. Entonces, a mí este tema que tienen algunas auxiliares, que tienen sus 45 años, que ya están bastante..., bueno, que la vida, pues, ya les ha dado unos cuantos palos y han sabido salirse con su vida, con su historia personal, con sus familias, pues yo esto lo valoro mucho. (Trabajadora Centro Residencial, gestora)

En segundo lugar, una mirada más precisa de la realidad hace emerger cualidades de este empleo que encajarían a la perfección con aquellas competencias que se espera deben tener personas con alta cualificación: responsabilidad, capacidad de decisión, autonomía, rapidez de reacción, empatía, capacidad de detección de necesidades. Son competencias exigidas tanto por las profesionales de la gestión como por los familiares, pero no son reconocidas para la cualificación formal de estos trabajos: 
Sobre todo, el servicio, aparte de dar una atención al usuario, el SAD es un chivo expiatorio para que haya alguien en ese domicilio, para saber qué pasa. Independientemente de que se hagan tareas de higiene y todo eso, nosotros, cuando se pone el servicio, es para saber qué pasa en ese domicilio. Entonces claro, tiene que ser una persona que sea receptiva, que sea..., que se fije en los detalles, que vea las cosas. No que vaya, pues mira, hago un plato de sopa y me voy. No, eso no es la función del SAD. El SAD es estar ahí, ver qué pasa, escuchar, ser receptora, ver qué puede pasar, qué problemáticas... Tienes que estar alerta. Más que la tarea en sí que realizan, eso es lo de menos. Para mí es lo de menos. Es más saber qué hay, qué no hay, qué necesidades tiene, a nivel de salud sobre todo, por ejemplo, control de medicación, cosas así. Quién entra en casa, quién no entra. Es más un poco detective - por así decirlo-, que no técnico. (Trabajadora $\mathrm{SAD}$, gestora)

Desde el punto de vista de la naturalización de las competencias, se observa que el imaginario de los cuidados, como una característica femenina, contribuye a desprofesionalizar las habilidades necesarias para una buena atención. En este sentido, aparece una dicotomía entre las competencias actitudinales (más presentes en la familia) y las competencias técnicas (más presentes en el ámbito profesional) que invisibiliza las competencias aptitudinales de mediación, comunicación y gestión. La naturalización existente en los círculos informales acentúa el peso de los lazos familiares. Así, la competencia aptitudinal se convierte en la construcción de lazos de cariño y amistad, enmascarando esas competencias aptitudinales que requiere el buen desempeño del trabajo de cuidados:

Ya nos cogió más cariño, ya no estaba cascarrabias, le hacíamos las bromas, con cariñitos. Mi hermana, la que lo cuidaba por la noche, también es cariñosa, también le hacíamos los mimitos, porque yo creo que veíamos el cariño paternal de papá que dejamos en casa. Entonces, tú [lo] buscas. Y a mi hermana, le pasó igual. Yo le decía: "tú eres como mi padre". Claro que él era blanco, colorado, y mi papá era moreno, pero... (ríe). Entonces, yo le decía: “usted es como mi papá”. Y entonces él comienza a sentir el cariño, ¿no? (Empleada en el hogar)

En los círculos profesionales, esta naturalización se esconde tras la idea de un trabajo vocacional, mientras que entre las trabajadoras de los servicios, se apela a la necesidad de disfrutar con el trabajo, argumentando que la dureza que lo caracteriza sólo se soporta si reporta alguna satisfacción:

Sí, me gusta. Porque para aguantar y hacer los cambios de pañales, que hay variedad de cosas que se ven, verles las úlceras en los culos, las situaciones son duras, $y$ a veces se te mueren en tus brazos... Entonces, eso es duro, porque llegas a cogerles mucho cariño. (Trabajadora centro residencial, atención directa)

A mí, sí. Si no, no estaría. Yo no pensaba que me gustara tanto. No, sí que me gusta, mucho, ¿eh? Además, me lo paso bien con ellos. Y a lo mejor están aquí y están pintando, o lo que sea, y sale una música y me pongo a bailar. (Trabajadora Centro de Día, atención directa)

En cualquier caso, la visión más generalizada sería la construcción de una profesional, con sentimiento de grupo y vocación para el puesto de trabajo. Eso recuerda a la idea de segmento profesional y la necesidad de articular una cierta estructura de poder capaz de regular las puertas de acceso a la profesión. Un panorama que a priori parece estar lejos de la atención directa. Asimismo, es necesario resaltar la contradicción que se produce en torno a los conocimientos de las gestoras del cuidado. En su mayoría,son diplomadas universitarias, por tanto, con conocimientos reconocidos y con cierto prestigio. Sin embargo, para la práctica cotidiana de su trabajo, suelen manifestar que los estudios universitarios no las preparan para tratar personas mayores, una preparación que sí tienen las trabajadoras auxiliares:

Es que el problema es que la enseñanza universitaria es muy genérica, es muy básica, y no te prepara. No te prepara para una tarea en concreto, porque después, en el tema de trabajo social, es tan amplio el abanico de lugares donde puedes tú trabajar, que claro, no puede ser muy específica, tampoco, la carrera. 0 sea, si ahora estuviera trabajando en la prisión o estuviera trabajando en una escuela. (Trabajadora Centro de Día, gestora)

Por otro lado, las mismas gestoras reconocen que aquello más importante para el buen desempeño de su trabajo es saber cuidar, saber relacionarse con las personas. De ahí la contradicción: la formación tiene un reconocimiento formal, pero se necesitan cualidades que no se aprenden en esa formación reglada. Unas cualidades que, cuando las que las tienen son las trabajadoras de la atención directa, no tienen valor ni prestigio:

Lo que más me gusta es, o sea, cuando puedo estar sin presión con ellos, haciendo. Haciendo actividades, el programa. No me gusta tanto la programación, pero me gusta más lo, ya, llevar a cabo la actividad, o sea, hacer. Hacer las excursiones con ellos, hablar con ellos, y ver lo que disfrutan, lo que no disfrutan. Esto es lo que más me gusta. Tener, tener tiempo sin presiones para hablar, para escucharlos, porque te expliquen sus historias. Es lo más, es lo más bonito de todo. (Trabajadora Centro de Día, gestora)

En definitiva, a partir de los análisis del discurso, emergen dos cuestiones fundamentales: la dificultad para explicar qué es un buen cuidado, incluso 
para las propias profesionales, y la evidencia de las relaciones de poder que guarda el trabajo de cuidar a las personas. Estas dos cuestiones están intimamente relacionadas con las dificultades de prestigiar el sector de los cuidados de larga duración. Un sector donde existe una jerarquía entre las profesiones ya existentes que afecta al contenido y desarrollo de las tareas que se ven obligadas a desempeñar las actuales empleadas de los servicios. El tipo de trabajo (contenido) influye en las exigencias formativas y en el reconocimiento de las cualificaciones profesionales, al estar ligado a los imaginarios sobre el subestimado trabajo doméstico y familiar. Así se entiende el proceso no neutral, desde la perspectiva de género, de la construcción de la cualificación profesional que no incorpora determinados saberes que son imprescindibles para el desempeño de las tareas de cuidado y que, al contrario de lo que está aceptado socialmente, no todo el mundo adquiere. Asimismo, los resultados ponen de manifiesto la importancia del espacio donde tienen lugar las tareas de cuidados como una dimensión explicativa de las dificultades de profesionalización. Un espacio en el que se producen relaciones distintas entre quien presta, quien recibe y quien encarga los cuidados.

\section{Conclusiones}

A lo largo del texto, se han planteado algunos de los principales debates sobre la profesionalización en el sector de los cuidados de larga duración en España. El artículo se inicia con un recorrido sobre el olvido de este sector en el nacimiento y desarrollo del Estado del bienestar, en parte porque el pacto fundacional se erigió sobre la aceptación de la división sexual del trabajo. Sin embargo, el envejecimiento de la población europea ha supuesto un reto para los Estados, que deberán atender las necesidades de cuidados de larga duración de una forma u otra. En España, parece claro que el régimen de cuidados sigue muy anclado en un modelo familiar como eje central desde donde resolver las necesidades de cuidados. 0 lo que es lo mismo, que son las familias, y especialmente las mujeres adultas de las familias, quienes se encargan de pensar, resolver y, en gran medida, ejecutar los cuidados de larga duración.

El objetivo del artículo ha sido analizar los límites que dificultan la profesionalización del trabajo de cuidados de larga duración teniendo en cuenta el contexto institucional del sistema de cuidados español. El trabajo de campo ha permitido hallar algunas respuestas, distinguiéndose dos núcleos importantes de dificultades que hacen difícil desarrollar una estrategia de cualificación de los trabajos: factores relacionados con los imaginarios sociales sobre el contenido del cuidado y sobre quién debe prestarlo; y factores que ligados al reconocimiento de las cualificaciones y las estructuras jerárquicas dentro de la profesión.
La dificultad de definir el cuidado, las exigencias de quién decide cómo se cuida, la individualización del cuidado, la preferencia por el espacio del hogar, no parecen ser buenos aliadas para emprender la construcción de un segmento cualificado. En este sentido, se ha explicitado que los imaginarios del cuidado familiar pesan mucho y dificultan que estos empleos sean vistos como algo profesional. Esto significa que los imaginarios sobre el cuidado son compartidos por familiares que cuidan o gestionan el cuidado, trabajadoras del hogar, trabajadoras en las instituciones e incluso por parte de los profesionales que tienen cierta capacidad de incidencia en el diseño del sistema de atención a las personas. La persistencia de un modelo familista propio de un país mediterráneo limita la capacidad de avanzar hacia sistemas de profesionalización. En este sentido, del análisis del discurso de las personas entrevistadas emerge una cultura de los cuidados familista y un ideal de cuidados donde predomina el deseo de ser cuidado "en casa y con la familia". A pesar del reconocimiento legal de la necesidad de profesionalización que supone la LAPAD, persisten límites que hacen difícil la construcción de un sistema profesionalizador en el sector de los cuidados de larga duración. La persistencia de la división sexual del trabajo se traduce en un imaginario colectivo sobre el cuidado y su vinculación exclusiva al ámbito privado y del hogar. Los saberes, aptitudes, y conocimientos que requiere el desempeño de estas actividades parecen continuar muy impregnados de esos imaginarios colectivos que son especialmente fuertes en una sociedad familista como la española.

Por otro lado, la cualificación necesaria para el buen desempeño del trabajo de cuidados no logra convertirse en cualificación formalmente reconocida. Esto apunta a que el propio contenido del trabajo, un trabajo socialmente vinculado al trabajo doméstico y familiar, femenino e invisible, explica las dificultades para prestigiar los saberes relacionados con el desempeño del trabajo de cuidados de larga duración. La construcción de la cualificación profesional se realiza bajo esos supuestos y es asumida por la mayoría de profesionales, quienes naturalizan las habilidades aprendidas y las capacidades necesarias para desempeñar con calidad el trabajo de atención a las personas. No se valoran los saberes necesarios para el buen desarrollo de una actividad de estas características, no se tienen en cuenta aquellos saberes indispensables para la generación del bienestar de la persona cuidada, algo de lo que son muy conscientes las propias trabajadoras. Aunque las tareas que conlleva el trabajo de cuidados en la atención directa (cuidado físico y continuado) pueden conllevar procesos de capacitación técnica que conduzcan a unas mejores condiciones de empleo y un mayor reconocimiento del trabajo, lo que parece entreverse es la persistencia de la desigualdad y la justificación de la estructura jerárquica entre profesionales. En este sentido, resulta difícil pensar en estrategias para prestigiar los empleos relacionados con el cuidado 
cotidiano de las personas. Se observa que el ideal de "en casa y en familia", que entiende que el buen cuidado es el que prestan las mujeres adultas de las familias, traspasa los procesos de construcción de la profesión y dificulta que las propias profesionales se imaginen como empleadas cualificadas.

Este elemento también genera una diferencia y desigualdad clara entre perfiles profesionales, es decir, entre las personas que trabajan en la atención directa (tareas directamente relacionadas con el cuidado personal) y las personas que desempeñan tareas de gestión (tareas de organización de los cuidados en el ámbito profesional).

Por el contrario, recuperando alguno de los debates teóricos expuestos, aquello que parece más claro es que, dada la estructura del sector y sus condiciones, va a ser difícil hacer emerger estrategias profesionales sólidas, capaces de articular una demanda clara de la profesionalización del sector. El análisis de las percepciones subjetivas de las profesionales y empleadas entrevistadas nos indica que la profesionalización está lejos de poder paliarse reformulando la acreditación de las habilidades y competencias actualmente transmitidas en la formación requerida. Finalmente, cabe subrayar que las demandas de individualización del cuidado contribuyen a invisibilizar la relación conflictiva entre quien presta y quien recibe: se antepone la necesidad de unos, ocultando las relaciones de poder y las desigualdades que conlleva el trabajo de cuidados cuando no se percibe como una profesión. 


\section{Bibliografía}

ABBOT, A. (1988): The System of Professions. Essay on the Division of Expert Labour, Londres, University of Chicago Press.

ANXO, D.; y FAGAN, C. (2005): “The family, the state and now the market: The organisation of employment and working time in home services for the elderly", en BOSCH, G.; y LEHNDORFF, S. (eds.) Working in the Service Sector. A Tale from Different Worlds, Londres, Routledge.

BETTIO, F.; y VERASCHAGINA, A. (coord.) (2012): Long-Term Care for the Elderly. Provisions and Providers in 33 European Countries, Luxemburgo, Comisión Europea [/http://ec.europa.eu/justice/genderequality/files/elderly_care_en.pdfs].

CAMERON, C.; y MOSS, P. (2007): Care Work in Europe. Current Understandings and Future Directions, Londres, Routledge.

CARRASCO, C.; BORDERÍAS, C.; y TORNS, T. (2011): El trabajo de cuidados. Historia, teoría y políticas, Madrid, Catarata.

CRISTOPHERSON, S. (1997): Childcare and Elderly Care: What Occupational Opportunities for Women?, serie OCDE Labour Market and Social Policy Occasional Papers, n-27, OECD Publishing [〈http://dx.doi.org/10.1787/047834852710〉].

CROMPTON, R.; y LE FEURVE, N. (2003): “Continuity and change in the gender segregation of the medical profession in Britain and France", International Journal of Sociology and Social Policy, vol. 23, n-4-5, págs. 36-58.

DAVIES, C. (1996): "The sociology of professions and the profession of gender", Sociology, vol. $30, n^{0}-4$, págs. $661-78$

DEVETTER, F.; y ROSSEAU, S. (2007): “Services domestiques: quelles perspectives pour une strategié d'industrialisation?", Revue d'Économie Industrielle, vol. 119, $\mathrm{n}^{\circ}-3$.

DEVETTER, F. X. et al. (2008): L'Aide à domicile face aux services a la personne. Mutations, Confusions, paradoxes, Lille, Centre Lillois d'Études et de Recherches Sociologiques et Économiques.

DUSSUET, A. (2002): “Le genre de l'emploi de proximité”, Lien Social et Politiques, no-47, págs. 143-154.

DUSSUET, A. (2005): Travaux des femmes, enquêtes sur les services à domicile, París, L'Harmattan.

DUYVENDAK, J. W.; KNIJN, T.; y KREMER, M. (2005): Policy, People, and The New Professional. Deprofessionalisation and Re-profesionalisation in Care and Welfare, Ámsterdam, Amsterdam University Press.

ESPAÑA (2011): “Real Decreto 1620/2011, de 14 de noviembre, por el que se regula la relación laboral de carácter especial del servicio del hogar familiar", Boletín Oficial del Estado, no 277, 17-11-11, págs. 119.046119.057 [shttp://www.boe.es/buscar/doc. php?id=BOE-A-2011-17975'].

- (2006): “Ley 39/2006, de 14 de diciembre, de Promoción de la Autonomía Personal y Atención a las Personas en Situación de Dependencia”, Boletín Oficial del Estado, n-0 299, 15-12-06, págs. 44.142-44.156 [<http://www.boe.es/ buscar/doc.php?id=BOE-A-2006-21990〉].

- (1985): “Real Decreto 1424/1985, de 1 de agosto, por el que se regula la relación laboral de carácter especial del Servicio del Hogar Familiar", Boletín Oficial del Estado, no 193, 13-8-85, págs. 25.617-25.618 [khttp://www.boe.es/ buscar/doc.php?id=BOE-A-1985-17108>].

EVERTSSON, L. (2000): “The Swedish welfare state and the emergence of female welfare state 
occupations", Gender, Work and Organisations, vol. 7, nํㅜ 4, págs. 230-241.

FRAISSE, G. (2000): "Servidumbre, servicios de proximidad y democracia", en M. MARUANI, M.; ROGERAT, C.; y. TORNS, T. (eds.), Las nuevas fronteras de la desigualdad, Barcelona, Icaria.

GADREY, J. (1994): “La modernisation des services professionels. Rationalisation industrielle ou rationalisation professionelle?", Revue Française de Sociologie, vol. 35, no 2 , págs. 163-195.

HEBSON; G.; RUBERY, J.; y GRIMSHAW, D. (2015): "Rethinking job satisfaction in care work: Looking beyond the care debates", Work, Employment and Society, vol. 29, $\mathrm{n}$ - 2, págs. 314-330.

HENRIKSSON, L.; WREDE, S.; y BURAU, V. (2006): "Understanding professional projects in welfare service work: Revival of old professionalism?", Gender, Work and Organization, vol. 13, nㅡ 2, págs. 174-192.

HUGMAN, R. (1991): Power in Caring Professions, Basingstoke, MacMmillan.

INSTITUTO DE MAYORES Y SERVICIOS SOCIALES (2005): Atención a las personas en situación de dependencia en España: libro blanco, Madrid, Imserso [shttp://www.dependencia.imserso. es/InterPresent2/groups/imserso/documents/ binario/libroblanco.pdf〉].

JANY-CATRICE, F.; PUISSANT, E.; y RIBAULT, T. (2009): "Associations d'aide à domicile: pluralité des héritages, pluralité des professionnalités", Formation Emploi, vol. 107, n- 3, págs. 77-91.

KNIJN, T.; y VERHAGEN, S. (2007): “Contested professionalism payments for care and the quality of home care", Administration and Society, $\mathrm{n}-39$, págs. 451-474.

KRÜGER, K.; y JIMÉNEZ, E. (2013): “La Ley de Dependencia, ¿un cambio en el régimen de bienestar español? Familiarización versus profesionalización”, Scripta Nova. Revista Electrónica de Geografía y Ciencias Sociales, vol. XVII, nํ- 436 .

KUHLMANN, E.; y LYNN, I. (2008): “Gender, professions and public policy: New directions", Equal Opportunities International, vol. 27, no 1 , págs. 5-18.
LEITNER, S. (2003): "Varieties of familism. The caring function of family in comparative perspective", European Societies, vol. 5, no 4, págs. 353-375.

LYON, D.; y GLUKSMANN, M. (2008): “Comparative configurations of care work across Europe", Sociology, vol. 42, noำ págs. 101-118.

MARTÍNEZ BUJÁN, R. (2011): “La reorganización de los cuidados familiares en un contexto de migraciones internacional", Cuadernos de Relaciones Laborales, vol. 28, ํㅡ1, págs. 93-123.

MILLER, D. A. (1981): “The 'sandwich' generation: Adult children of the aging", Social Work, septiembre, págs. 419-423.

PARELLA, S. (2003): Mujer, inmigrante y trabajadora: la triple discriminación, Barcelona, Anthropos.

PICKARD, L. (2012): "Substitution between formal and informal care: A natural experiment in social policy in Britain between 1985 and 2000", Ageing and Society, n-0 32, págs. 147-175.

RECIO, A. (1997): Trabajo, personas, mercados: manual de economía laboral, Barcelona, Icària.

RIBAULT, T. (2008): “Aide à domicile: de l'idéologie de la professionnalisation à la pluralité des professionnalités”, Revue Française de Socioéconomie, vol. 2, nํㅡ 2, págs. 99-117.

RIBAULT, T. (2009): La qualité de l'emploi dans les services à la personne: le cas français, Working Papers. Serie Recherche, $\mathrm{n}-1$, Institut de Recherche de la Maison Franco-Japonaise.

SARACENO, C. (1995): “Familismo ambivalente y clientelismo categórico en el Estado del bienestar italiano", en SARASA, S.; y MORENO, L. (eds.), El Estado del bienestar en la Europa del Sur, Madrid, CSIC.

SARACENO, C. (2010): "Social inequalities in facing old-age dependency: A bi-generational perspective", Journal of European Social Policy, nํㅜㄹ, págs. 32-44.

TORNS, T. et al. (2014): Nuevas profesiones para la organización social del cuidado. [<http://www. inmujer.gob.es/areasTematicas/estudios/ estudioslinea2014/docs/Nuevas_profesiones_ organizacion_social.pdf>].

WILLIAMS, C. (2004): "The sandwich generation", Perspectives on Labour and Income, vol. 5, ํㅜ 9, págs. 5-12. 